\title{
68. Vibration and Strength of Turbine Blade. VII Reviewing the Foregoing Notes
}

By Akimasa ONo, M.J.A.

(Comm. May 19, 1960)

On reviewing the foregoing Notes I to VI so far issued, it seems that they have to be summarized and supplemented considering application, as will be made in the following beginning with resuming the topics.

\section{Topics Resumed}

The topics hitherto taken up form three groups. First, Notes I and II are on natural frequency of a bar, resonance vibration, and vibration stress. Thereby comes the clamp-condition or more exactly the elasticity-effect of clamping on frequency and stress into question, and this particularly with regard to c.-free bar (written for clampedfree-) vibrating in the gravest mode.

This elasticity-effect is considered specially in Notes III and IV, of which the first one is concerned with exact calculation made on uniform bar, and the second with approximate calculation made for showing the basis underlying the previous calculation. Both are on c.-free bar except a passage (of Note III) particularly on the clamped end of c.-supported bar (written for clamped-supported-).

Lastly follow Notes V and VI on the elasticity-effect existing at both ends of c.-supported bar. The first one is again on uniform bar viewed by means of exact calculation, and the second gives approximate calculation - both regarding the gravest mode. As the latter calculation is on c.-supported bar of any arbitrary end-conditions including even what may be thought for c.-free bar, Note VI covers, so to speak, Note IV.

On the whole the investigation aims at finding the vibration stress with due attention to the elasticity-effect of the end or ends - an effect caused by the conditions deviating more or less from certain standard conditions taken for reference. This being so, the selection of standard conditions can be made quite freely with the approximate calculation last mentioned. Remembering this, we will recapitulate some formulae in thought of application.

\section{Vibration Stress-Summary of Calculation}

Let us resume with

$\sigma_{r}=$ the vibration stress (amplitude of alternating stress) in resonance vibration caused by a harmonic force-component $\nu f_{0}$ per unit length of blade, length $l, f_{0}$ denoting the mean intensity of force, 
$\sigma_{0}=$ the static stress due to the bending moment $f_{0} l^{2} / 2$,

$N^{\prime}=$ a numerical factor, which is proportional directly to the integral of the eigen-function $y$, written $(y)$, and inversely to the logarithmic decrement $\delta$ of damping.

Then

$$
\sigma_{r}=\sigma_{0} 2 \nu N^{\prime},
$$

and $N^{\prime}$ is expressible in relation with a standard value of it, $N$, e.g. in an approximate formula as

$$
N^{\prime}=N v^{2} \quad \text { with } \quad v=p_{\text {th }} / p_{\text {obs }},
$$

$v$ being the ratio of the frequencies, $p_{\text {th }}$ and $p_{\text {obs }}$, theoretical and observed. The relation in (2) is originally for c.-free bar being first introduced in Note II. It wants considering more generally, as occurs below.

By way of extending our view we have now recourse to Note VI, and write the eigen-function $y$ of the gravest mode

$$
y=y_{1}+\alpha x
$$

approximately, $y_{1}$ representing a standard eigen-function, from which $y$ differs by $\alpha x$, namely, the inclination multiplied by the distance measured from the clamped end. On integrating (3) over the length $l$,

$$
(y)=\left(y_{1}\right)+\alpha l^{2} / 2 \text {, }
$$

which expression enters (1) as a factor in $N^{\prime}$ marking the difference of $\sigma_{r}$ from the standard value, written $\sigma_{r}^{(1)}$.

In computing $\sigma_{r}$ in accordance with (3) $\delta$ is regarded as being a constant. To this point we will come back in the next article, but assuming so for the present we have a relation between $\sigma_{r}$ and $\sigma_{r}^{(1)}$ corresponding to $y$ and $y_{1}$, respectively, such that

$$
\sigma_{r}=\sigma_{r}^{(1)}\left(\frac{\alpha}{\left(y_{1}\right)} \frac{l^{2}}{2}+1\right),
$$

showing that $\sigma_{r}>\sigma_{r}^{(1)}$.

Further calculation in the text refers to uniform section; it appears, however, that (4) can be written generally in the form:

$$
\sigma_{r}=\sigma_{r}^{(1)}\left[\text { const. }\left(v^{2}-1\right)+1\right] \text {. }
$$

Moreover, putting $\sigma_{r}=\sigma_{r}^{(1)} N^{\prime} / N$,

$$
N^{\prime}=N\left[\text { const. }\left(v^{2}-1\right)+1\right]=\sim N v^{2} \text {. }
$$

The const. in (5) depends upon $y_{1}$ as well as upon the cross-sectional area if this varies, being a little greater than unity in a special case of uniform bar. Apart from an exact thing, the approximation made in the last expression will not be inadmissible provided $v$ is limited to a small range over 1 , e.g. $v^{2}=1-1.1$ in view of the special case mentioned above. Anyhow, it is better to take the const. fully. 


\section{Variation in Damping}

Before going into this subject the foregoing remark on $\delta$ wants elucidation. In fact the internal friction of blade material is taken at the outset as a source of damping in accounting for $\delta$, understanding adjusting it to comply with experience, as can be made by experimenting blades in some way. By this reason $\delta$ is thought first to be proportional to strain, that is, to $y_{x x}$ or $y_{1 x x}$ according to (3), giving rise to no difference between the two; so $\delta$ is taken as being a constant in the approximate calculation.

This assumption is, strictly speaking, inaccurate, as $y_{x x}$ is not exactly equal to $y_{1 x . x}$ because of (3) being simplified by neglecting functions higher than the first, $y_{1}$. Therefore, needless to say that $\delta$ is liable to vary on changing from $y_{1}$ to $y$ though slightly.

Apart from $\delta$ as such, the aspect of it is quite different in blade set loosely on rotor as is current in gas-turbine. Of loose blading $\delta$ owes its origin greatly to sliding friction whose effect has to be studied separately from internal friction, and above all specifically according to form of root, but in effect $\delta$ is known to vary with running speed. Beginning with a low speed, where the loose blade-end slides against the groove-surface in contact resulting in a great value of $\delta$, this decreases with the rise of speed until it takes finally a value, which betrays the tightness being brought about by the action of the centrifugal force. In this state the blade behaves itself seemingly as being a uisual c.-free bar.

In any way a great value of $\delta$ is attractive and perhaps misleading too. The true merit of it has to be decided by considering the direct effect of $\alpha$ at the same time. To make this we have to multiply the formulae (4) et seq. by a factor $\delta / \delta^{\prime}$ distinguishing $\delta^{\prime}$ for loose blade from $\delta$ for tight one. Whether $\sigma_{r}$ in loose blade may be smaller than $\sigma_{r}^{(1)}$ in tight one, or $N^{\prime}$ be less than $N$, depends upon the relative values of the two factors taken together; one factor may or may not weigh so much as the other.

A similar consideration will be necessary even for the elasticityeffect, if small variation aforesaid is wanted to be taken into account, a rough measure of the criterion being $v^{2}\left(\delta / \delta^{\prime}\right)$.

\section{Strength of Shrouded Blades}

Under this topic which is proper to most steam-turbines come, speaking roughly, the vibrations of both types, c.-free and c.-supported. Now turning to (3), it is to be remembered that $y_{1}$ can be chosen freely as to the end-conditions - even within the category of c.-supported; so we can take it for representing an ideal, standard state thought for blades of a system, and compare another $y$ assumed really in an actual state. Then $N^{\prime}$ bears a ratio to $N$ as given by $v^{2}$ approximately. 
Provided $v$ is given, $\sigma_{r}$ can be compared with $\sigma_{r}^{(1)}$ accordingly.

This consideration gives ground to the process pursued in Note II with regard to two broken blades except that they are taken rather tentatively as a c.-free bar.

To restate the process in brief, we begin with finding $N^{\prime} / N$ through $v^{2}$, preferably taking the const. in (5) fully as it is computed. Then we determine $\nu N^{\prime}$ by identification of $\sigma_{r}$ in (1) with a value of it obtainable from the endurance limit of the blade material corrected for stress-concentration and for action of the static stress. Whether $\nu N^{\prime}$ found in this way may belong to one or the other type of vibrations is a matter relating to the frequency of the force-component. In general c.-free refers probably to the peripheral conditions (of flowing medium) at large, while c.-supported to more minute conditions (e.g. the distribution of nozzles). This is stated reservedly in general terms in absence of concrete examples.

After all $\nu N^{\prime}$ together with $N^{\prime} / N$ gives $\nu N$, and then $\nu$ and $N$ if we know or assume either one. The value of $\nu N$ thus found is obviously subject to the variation due to the scattering of strength figures, yet it must be more regular than $\nu N^{\prime}$.

Returning to $N^{\prime} / N$ as an item taken for defining the stress-ratio, the determination of $v$ calls special attention. As values of $v$ may be scattered among blades bound together, a correct value proper to a particular blade must be presumably difficult to be made known. Still, frequency-tests will give at least some information about $v$ in a sense. For it appears that simple tests on individual blades in a form of c.-free bar will open a way for grasping the general feature and eventually meeting with any special case as breaking, since scattering of the values found from a number of blades tested in similar conditions will reveal the accuracy of work. Therefore, the frequency-testprobably a little more than average routine test in engineering laboratory - will well deserve undertaking.

The above remark is inconclusive, but it may not sound, I hope, so unpractical as thinking fantastically of wandering the world through to find something, say, the dot over $i$ (das Tüpfchen auf das $i$ ). 